\title{
Acquired resistance to innate immune clearance promotes Klebsiella pneumoniae ST258 pulmonary infection
}

\author{
Danielle Ahn, ${ }^{1}$ Hernán Peñaloza, ${ }^{2,3}$ Zheng Wang, ${ }^{4}$ Matthew Wickersham, ${ }^{1}$ Dane Parker, ${ }^{1}$ Purvi Patel, \\ Antonius Koller, ${ }^{5}$ Emily I. Chen, ${ }^{5,6}$ Susan M. Bueno, ${ }^{2,3}$ Anne-Catrin Uhlemann, ${ }^{4}$ Alice Prince ${ }^{1,6}$ \\ 'Department of Pediatrics, Columbia University Medical Center, New York, New York, USA. ${ }^{2}$ Millennium Institute on \\ Immunology and Immunotherapy, Santiago, Chile. ${ }^{3}$ Departamento de Genética Molecular y Microbiología, Facultad \\ de Ciencias Biológicas, Pontificia Universidad Católica de Chile, Santiago, Chile. ${ }^{4}$ Department of Medicine, Columbia \\ University Medical Center, New York, New York, USA. ${ }^{5}$ Proteomics Shared Resource at the Herbert Irving Comprehensive \\ Cancer Center, Columbia University Medical Center, New York, New York, USA. ${ }^{6}$ Department of Pharmacology, Columbia \\ University Medical Center, New York, New York, USA.
}

Adaptive changes in the genome of a locally predominant clinical isolate of the multidrugresistant Klebsiella pneumoniae ST258 (KP35) were identified and help to explain the selection of this strain as a successful pulmonary pathogen. The acquisition of 4 new ortholog groups, including an arginine transporter, enabled KP35 to outcompete related ST258 strains lacking these genes. KP35 infection elicited a monocytic response, dominated by Ly6C ${ }^{\text {hi }}$ monocytic myeloidderived suppressor cells that lacked phagocytic capabilities, expressed IL-10, arginase, and antiinflammatory surface markers. In comparison with other $K$. pneumoniae strains, KP35 induced global changes in the phagocytic response identified with proteomics, including evasion of $\mathrm{Ca}^{2+}$ and calpain activation necessary for phagocytic killing, confirmed in functional studies with neutrophils. This comprehensive analysis of an ST258 $K$. pneumoniae isolate reveals ongoing genetic adaptation to host microenvironments and innate immune clearance mechanisms that complements its repertoire of antimicrobial resistance genes and facilitates persistence in the lung.

Authorship note: A.C. Uhlemann and A. Prince are co-senior authors.

Conflict of interest: The authors have declared that no conflict of interest exists.

Submitted: July 21, 2016 Accepted: September 8, 2016 Published: October 20, 2016

Reference information: JCl Insight. 2016;1(17):e89704. doi:10.1172/jci.insight.89704.

\section{Introduction}

Carbapenem-resistant Klebsiella pneumoniae (CRKP) sequence type 258 (ST258) is a major cause of health care-associated infections worldwide $(1,2)$ such as ventilator-associated pneumonia and catheter-related blood stream infections. These are major complications in the critically ill, prolonging length of stay, incurring additional medical costs, and leading to death in the majority of cases $(3,4)$. ST258 is the most common sequence type of CRKP in the United States, accounting for $70 \%$ of cases in the national repository (5). These bacteria readily undergo recombination events and have highly variable plasmid content, antimicrobial resistance patterns, and capsular composition (6). CRKP ST258 consists of at least 2 distinct clades, suggesting the active selection of evolutionarily advantageous isolates (7), unlikely to be attributed to their antibiotic resistance alone.

In parallel with their adaptation to antimicrobial exposure, these opportunists have evolved mechanisms to evade host innate immune clearance. Although $K$. pneumoniae is typically considered as a single entity, even among the ST258 strains very different patterns of infection are elicited in model systems (8). Despite associated mortality rates of up to $50 \%$, the CRKP strains isolated from recent outbreaks appear to be less virulent in model systems than the prototypical $K$. pneumoniae serotype 2 reference strain ATCC 43816 (KPPR1) (9). In contrast with other opportunists such as Pseudomonas aeruginosa, CRKP expresses few toxins. It is relatively resistant to neutrophil-mediated clearance (10), but instead is phagocytosed primarily by inflammatory monocytes (11). This is in contrast with the better-studied and much more virulent KPPR1 strain, which is eradicated through IL-17 signaling and neutrophil recruitment (11-13).

The importance of monocyte populations in the effective host response to $K$. pneumoniae infection has been well recognized $(8,14)$. However, monocyte classification is not entirely straightforward because of the heterogeneity and plasticity of these cells in response to cytokines in the microenvironment. 
Monocytes adopt an immunosuppressive phenotype, the so-called monocytic myeloid-derived suppressor cells (M-MDSCs) in the context of high levels of GM-CSF, IL-6, CCL2, and other cytokines (15, 16). These monocytes promote expansion of Treg populations, contribute to the resolution of infection to prevent ongoing tissue damage, and provide an immunotolerant cytokine milieu.

In the experiments detailed in this report, we postulated that a locally predominant clone of ST258, isolate 35 (KP35), has actively adapted to the milieu of the human airway. KP35 has acquired 4 new ortholog groups (OGs), including an arginine transporter that enhances the ability of the organisms to persist in the lung. Its adaptation included the recruitment of an immunosuppressive M-MDSC population to the airway, lacking phagocytic capabilities themselves and suppressing the proinflammatory cytokine responses needed for bacterial clearance. Our data suggest that the acquisition of phenotypically silent but immunologically significant mutations as well as antimicrobial resistance genes are a major factor in the success of CRKP.

\section{Results}

Characteristics of human colonization and murine modeling of CRKP infection. Clinically significant infections with CRKP are typically identified in patients who have been exposed to multiple antimicrobial agents and often have significant underlying diseases (1). This is illustrated by the phenotypically heterogeneous $K$. pneumoniae strains that were isolated repeatedly from the airway and blood over a 3-month period of a nonneutropenic mechanically ventilated patient (Figure 1A). We used a murine model of pulmonary infection to characterize the innate immune response to ST258 isolate 35 (KP35), a CRKP isolate representative of many strains recovered from our hospital, expressing the most common cps (capsular protein) and $w z i$ (cps component) genotypes (1). Following intranasal delivery of a high inoculum (10 $\mathrm{CFU})$ to WT C57BL/6J mice, greater than $10^{4} \mathrm{CFU} / \mathrm{ml}$ were isolated from the lungs and airways, accompanied by high-grade bacteremia and weight loss over the 96-hour course of infection (Figure 1, B and C). Persistence of bacterial burden was evident with greater than $10^{4} \mathrm{CFU} / \mathrm{ml}$ present in the bronchoalveolar lavage fluid (BALF) at 7 days of infection (Figure 1D). KP35 caused diffuse bronchopneumonia, with peribronchial consolidation and collagen deposition (Figure 1, E-G). This response differed from that evoked by the KPPR1 strain, which caused lethality at a much lower inoculum $(17,18)$ (Supplemental Figure 1, A and B; supplemental material available online with this article; doi:10.1172/jci.insight.89704DS1), or even a recent ST258 isolate that was much more readily cleared in a pneumonia model (8).

KP35 induces a biphasic cytokine response. To better understand how KP35 was able to persist in the airway, we quantified cytokine production over the course of infection. There was an initial induction of proinflammatory cytokines in response to intranasal inoculation with $10^{8} \mathrm{CFU}$ of KP35 with high levels of TNF and KC/CXCL1 expression in bronchoalveolar lavage fluid (BALF) by 4 hours, and IL-6 and IL-1 $\beta$ by 24 hours (Figure 2, A-D). However, production of these proinflammatory cytokines was quickly attenuated. Expression of the granulocyte cytokines and chemokines GM-CSF, G-CSF, MIP-1 $\alpha /$ CCL3, and IL-17, high initially, also waned (Figure 2, E-H). At 48 hours, a second wave of cytokine expression ensued, consisting of substantial amounts of CCL2, IL-10, VEGF, and M-CSF (Figure 2, I-L). Induction of cytokines known to be critical in KPPR1 clearance such as IFN- $\gamma$ and IL-12(p70) $(19,20)$ were elevated only after 96 hours of infection (Supplemental Figure 1C). The early cytokine response to KP35 infection differed substantially from that elicited by the opportunistic gram-negative pathogen $P$. aeruginosa at a lower inoculum $\left(10^{7} \mathrm{CFU}\right)$, which induced greater than 50 -fold increases in expression of $\mathrm{TNF}, \mathrm{KC}$, and IL-6 at 24 hours, whereas KP35 evoked antiinflammatory cytokines such as IL-1ra, TIMP-1, and IL-10 (Supplemental Figure 1D).

Recruitment of monocytes in response to KP35. Given the importance of macrophages and monocytes in $K$. pneumoniae pathogenesis, we characterized these populations in the KP35-infected lung and airways (Supplemental Figure 2, A-C). The resident alveolar macrophage population $\left(\mathrm{CD} 45^{+}\right.$SiglecF $\left.\mathrm{F}^{+} \mathrm{CD} 11 \mathrm{~b}^{\text {lo-mid }}\right)$ declined steadily over the 4-day infection (Figure 3A and Supplemental Figure 2D). In contrast with the important role resident alveolar macrophages play in the clearance of KPPR1 infection (21), clodronate depletion of these macrophages did not alter clearance of KP35 from the lung (Supplemental Figure 3, A-C). They were replaced by 48 hours with a 2-log increase in the so-called M-MDSCs $\left(\mathrm{CD} 45^{+} \mathrm{CD} 11 \mathrm{~b}^{+} \mathrm{MHCII}^{\mathrm{lo}} \mathrm{Ly} 6 \mathrm{C}^{\mathrm{h}} \mathrm{Ly} 6\right.$ $\mathrm{G}^{\mathrm{lo}}$ ) (Figure 3A and Supplemental Figure 2D). These M-MDSCs are recruited by monocyte chemokines, proliferate locally, and contribute to an antiinflammatory milieu, best characterized in models of tumor growth (15). The remaining $\mathrm{CD} 11 \mathrm{~b}^{+}$population consisted of $\mathrm{CD} 45^{+} \mathrm{CD} 11 \mathrm{~b}^{+} \mathrm{MHCII}{ }^{\mathrm{lo}} \mathrm{Ly} 6 \mathrm{C}^{\mathrm{hi}} \mathrm{Ly} 6 \mathrm{G}^{\mathrm{hi}}$ cells, a 

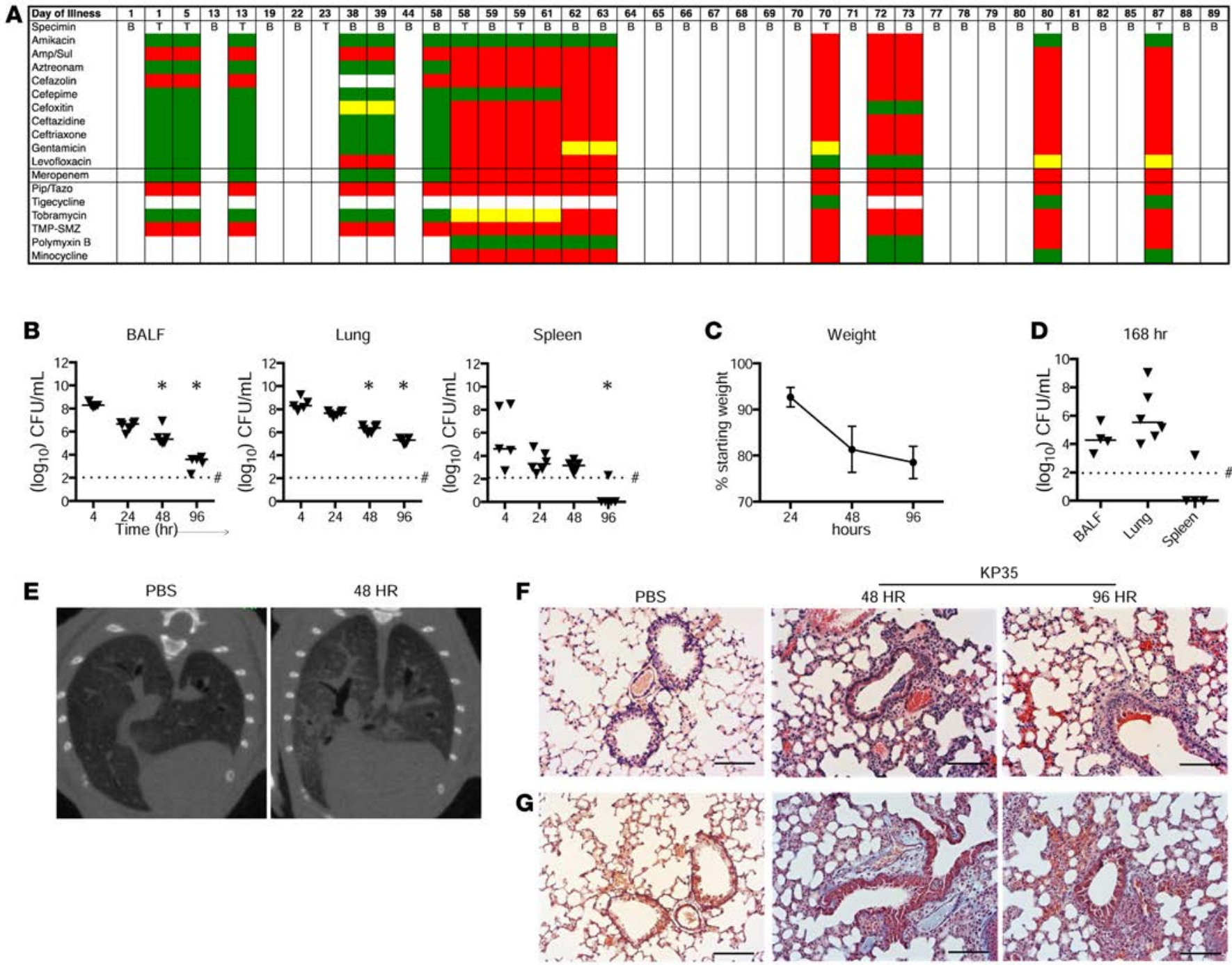

Figure 1. Kinetics of KP35 infection and host response. (A) Antimicrobial susceptibility of the $K$. pneumoniae strains isolated from the airway and bloodstream of a patient over a 3-month hospitalization. B, blood; T, tracheal aspirate. Red = resistant, Yellow = intermediate, or Green = sensitive minimum inhibitory concentration for the given isolate for the corresponding antibiotic. (B) Kinetics of KP35 clearance from bronchoalveolar lavage fluid (BALF), lung homogenate, and spleen following intranasal inoculation of $1 \times 10^{8}$ to $2 \times 10^{8} \mathrm{CFU}$ in WT mice over the course of a 4-day infection. \# = the lower limit of detection. Horizontal lines represent median values and each data point represents an individual mouse. $n=6$ per time point. All data were compiled from 2 independent experiments. $n=5-6$ per time point. ${ }^{*} P<0.05$, compared with CFU at 4 hours of infection, Kruskal-Wallis test, 1-way ANOVA, Dunn's correction for multiple comparisons. (C) Weight loss over the course of infection, data points represent mean values \pm SEM. $n=5-6$ per time point. (D) Bacterial load enumerated from selected compartments after 7 days of infection. \# = the lower limit of detection. Horizontal lines represent median values and each data point represents an individual mouse. $n=4-6$ per compartment. (E) CT imaging demonstrates diffuse bronchopneumonia 48 hours following KP35 infection. Representative axial images obtained with the Quantum FX CT Scanner in 3- $\mu$ m slices are shown. (F) Histopathology of KP35 pneumonia demonstrating peribronchial consolidation and cellular infiltrates in H\&E-stained sections of lung, PBS control, and KP35 infection at 48 and 96 hours. (G) Trichrome staining of KP35-infected lung sections demonstrating patchy disruption of the alveolar architecture and discrete areas of collagen deposition. Scale bars (F and $\mathbf{G}): 100 \mu \mathrm{m}$.

combination of inflammatory mononuclear cells that includes the granulocytic MDSCs (G-MDSCs), which can mature to become functional phagocytes and neutrophils (15) (Figure 3, B and C and Supplemental Figure 2D). Coincident with the influx of M-MDSCs we noted the abrupt suppression of STAT-1 signaling, a transcription factor that is usually critical in acute bacterial infection $(22,23)$. STAT-3 signaling, important for M-MDSC proliferation (24), was also induced following infection and was more sustained than STAT1 (Supplemental Figure 4, A-C). The prolonged recovery of CRKP from the airway indicated to us that the immunosuppressive properties of the M-MDSCs could be interfering with efficient bacterial eradication.

$M-M D S C s$ recruited in response to KP35 have an immunosuppressive phenotype. The M-MDSCs found in BALF at 48 hours after KP35 inoculation had not increased their display of the activation markers CD86 or MARCO 

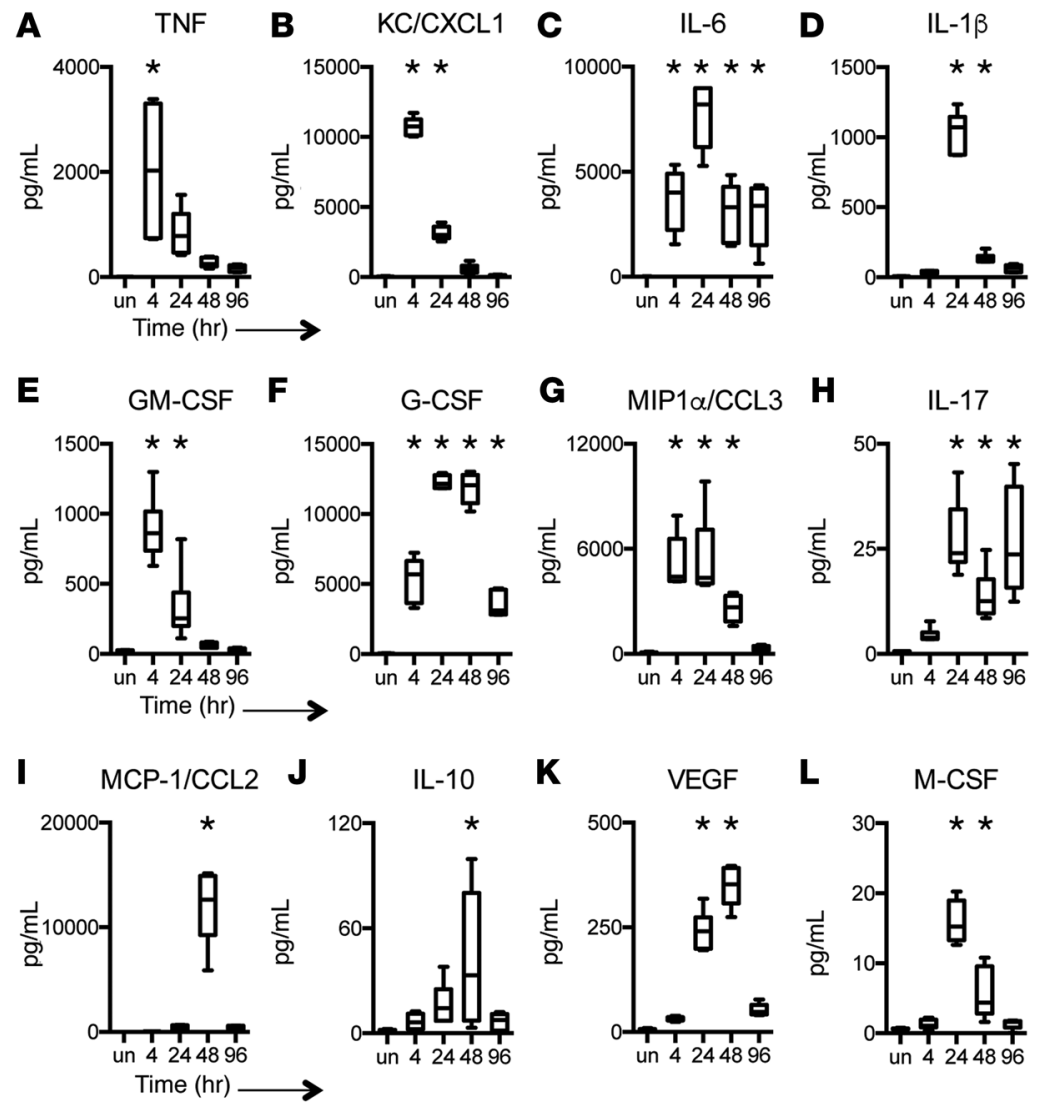

Figure 2. Biphasic cytokine response to KP35 infection. Selected cytokine and chemokine content of bronchoalveolar lavage fluid of WT mice following intranasal inoculation of $1 \times 10^{8}$ to $2 \times 10^{8}$ CFU in WT mice over the course of a 4-day infection was quantified by multiplex assay. ${ }^{*} P<0.05$, compared with uninfected (un) control, Kruskal-Wallis test, 1-way ANOVA, Dunn's correction for multiple comparisons. All data were compiled from 2 independent experiments, $n=6$ per time point. For data presented as box-andwhiskers plots, horizontal lines indicate the median, boxes indicate 25 th to 75 th percentiles, and whiskers indicate minimum and maximum values of the data set.

above the basal levels. However, they did have increased CD200R and CD206 at 48 hours after infection, surface markers associated with immunosuppressive phenotypes (Figure 3D and Supplemental Figure 2E). One explanation for the failure to activate monocytes could be the lack of KP35 immunogenicity, perhaps caused by changes in LPS or capsular polysaccharides.

However, KP35 readily induced TNF production in bone marrow-derived macrophages (BMD$\mathrm{Ms)}$ in a TLR4-dependent manner comparable with an $E$. coli LPS control, indicating that the monocyte response was not due to the inability of KP35 to activate canonical TLR4 signaling pathways (Figure 3E). Instead, it appeared more likely that KP35 was inducing an immunosuppressive response from the M-MDSC population.

To assess the contribution of the MDSC cytokine production to the immunostimulatory milieu of the KP35-infected airways, we measured gene expression of the $\mathrm{Ly}_{6} \mathrm{C}^{+}$leukocyte population harvested directly from the KP35-infected mice as compared with that elicited by E. coli LPS (Figure 3F and Supplemental Figure 5A). As expected, there was substantially less $\mathrm{KC}$ and TNF expression in $\mathrm{Ly}_{6 \mathrm{C}}{ }^{+}$cells harvested from BALF of KP35-infected mice than that induced by E. coli LPS. In contrast, significantly increased expression of arginase-1, iNOS, CCL2, IL-10, SOCS3, and Retnla (Fzz1) were quantified (Figure 3F), genes that are highly expressed by M-MDSCs. Despite lower absolute numbers of M-MDSCs in the infected airways, their cytokine products appear to determine the predominant immune response to KP35 infection.

KP35 is resistant to phagocytic killing by M-MDSCs. Inflammatory monocytes have been found to be important in the clearance of $K$. pneumoniae pulmonary infections (8). To assess the participation of the M-MDSC population in KP35 clearance, killing assays were performed in vitro using polarized M-MDSClike monocytes from WT murine BM. BM-derived MDSCs (BM/MDSCs) did not kill either KPPR1 or KP35 (Figure 4, A and B). This was in contrast with freshly harvested murine neutrophils, which killed greater than $99 \%$ of the KPPR1 inoculum within 30 minutes (Figure 4, C and D). Neutrophils had limited ability to kill KP35, as has been observed for other ST258 isolates (10). Moreover, KP35 and KPPR1 stimulated BM/MDSC products, which included expected surface markers and cytokines of M-MDSCs (Supplemental Figure 5, B-F), and actually suppressed the ability of murine neutrophils to kill KPPR1 (Figure 4, E and F). Thus, the M-MDSC population recruited in response to KP35 appears to impair bacterial clearance by failing to participate in phagocytic killing themselves and by producing cytokines that function to suppress neutrophil-mediated clearance.

To directly assess the importance of the M-MDSC population in blocking KP35 eradication, we attempted numerous selective depletion strategies, based upon work done in oncology to remove these cells and their inhibitory effects on immune-mediated tumor lysis (25) (Supplemental Figure 6). Using antiCCR2 and/or anti-TNFR2 to block accumulation of M-MDSCs $(8,26,27)$, we were unable to selectively deplete M-MDSCs without also affecting the G-MDSC/neutrophil populations.

KP35 induces global changes in the phagocytic response. Our data suggest that the typical proinflammatory immune signaling elicited by $K$. pneumoniae strains such as KPPR1 was not effective in the clearance of 

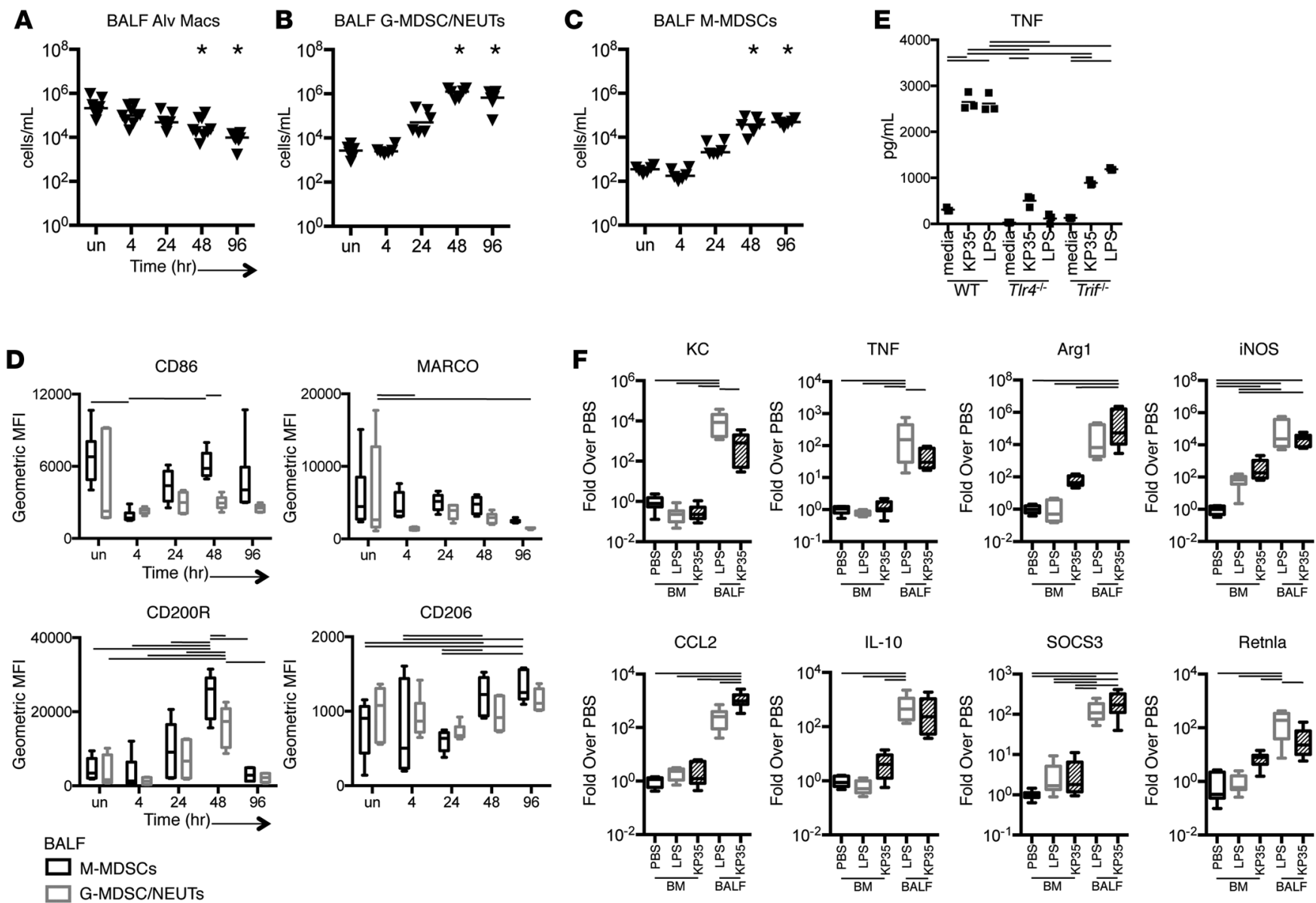

Figure 3. Recruitment of monocytes in response to KP35. (A-C) Cellular response to infection in bronchoalveolar lavage fluid (BALF) determined by flow cytometry - alveolar macrophages (Alv Macs) (CD45+SiglecF+CDIl11b $\left.{ }^{1-m i d}\right)$, granulocytic myeloid-derived suppressor cells/neutrophils (G-MDSCs/

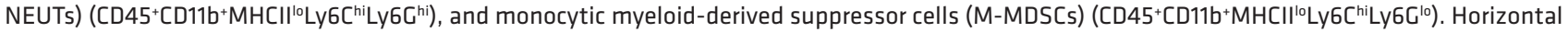
lines represent median values and each data point represents an individual mouse. All data were compiled from 2 independent experiments, $n=6$. ${ }^{*} P$ $<0.05$, compared with uninfected (un) control, Kruskal-Wallis test, 1-way ANOVA, Dunn's correction for multiple comparisons. (D) Changes in surface markers associated with M-MDSCs and G-MDSCs/NEUTs determined by geometric mean fluorescence intensity (MFI), $n=6$. For box-and-whiskers plots, horizontal lines indicate the median, boxes indicate 25th to 75th percentiles, and whiskers indicate minimum and maximum values of the data set. (E) Levels of TNF measured by ELISA from supernatants from immortalized bone marrow-derived macrophages (BMDMs) (WT, TIr4 $4^{-I^{-}}$, or Trif ${ }^{-1}$ ) incubated with KP35 or E. coli LPS $(10 \mu \mathrm{g} / \mathrm{ml})$ as a positive control for 4 hours. Representative graph of 2 independent experiments, $n=3$ per condition. (F) Cytokine and chemokine production by Ly6C ${ }^{+}$cells isolated from WT mice following exposure to KP35 $\left(1 \times 10^{8}\right.$ to $\left.2 \times 10^{8} \mathrm{CFU}\right)$ or E. coli LPS (50 $\mu$ g), measured by qRT-PCR compared with PBS control. $n=7$-9. For B-D, columns represent mean values \pm SEM, horizontal bars represent $P<0.05$ by 1 - or 2 -way ANOVA followed by Bonferroni's or Dunn's correction for multiple comparisons.

KP35. To better appreciate the host response to KP35, a proteomic analysis of BALF harvested from KP35and KPPR1-infected mice was performed. Pooled BALF recovered from KPPR1-infected (10 $\mathrm{CFU})$ and KP35-infected $\left(10^{8} \mathrm{CFU}\right)$ mice after 48 hours was analyzed to highlight differences in the host response. At this time point, mice were alive and equivalent inocula were recovered $\left(10^{6} \mathrm{CFU}\right)$. Both organisms activated infection-associated signaling pathways, acute-phase reactants, complement components, and proteins involved in inflammatory signaling (Figure 5A and Supplemental Table 5). Major differences were observed primarily in pathways important for chemotaxis and phagocytosis. Most striking were increases in components of actin cytoskeletal dynamics induced by KP35 (Figure 5B). Also prominently upregulated by KP35 were the $\mathrm{Ca}^{2+}$-binding proteins S100A9 (calprotectin) (51.68-fold) and thrombospondin (91.5-fold) (Figure 5C). S100A9 functions in $\mathrm{Ca}^{2+}$ sensing and cytoskeletal rearrangements during neutrophil transendothelial migration (28) and is a potent MDSC chemoattractant (29). Thrombospondin is involved in actin-based cellular dynamics important in inhibiting neutrophil function $(30,31)$. Proteins involved in calpain activation, a $\mathrm{Ca}^{2+}$-dependent protease critical for phagocytosis (32), were suppressed to a much greater degree by KP35, consistent with the finding that this strain is resistant to phagocytic killing (Figure 5D). 
A

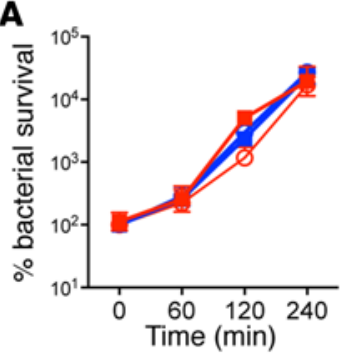

C

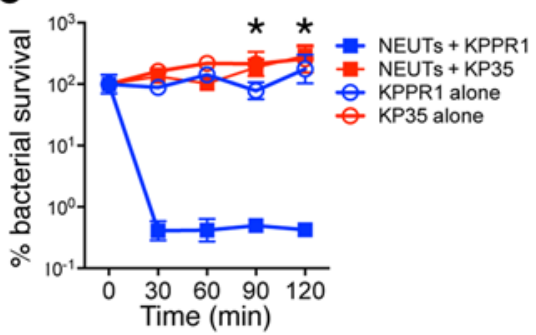

E

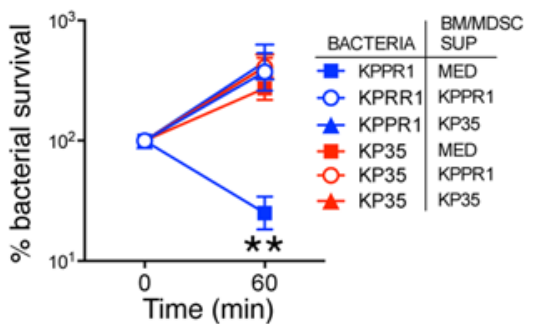

B

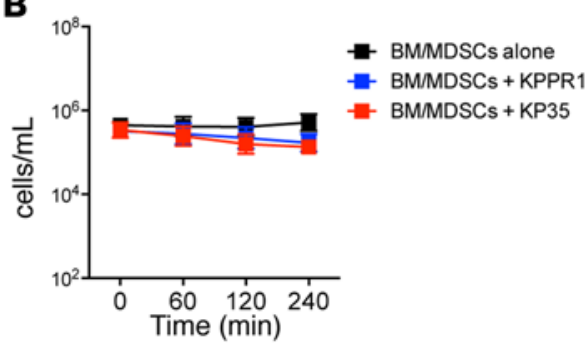

D

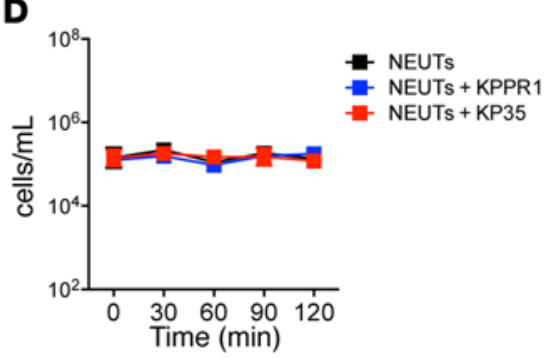

$\mathbf{F}$

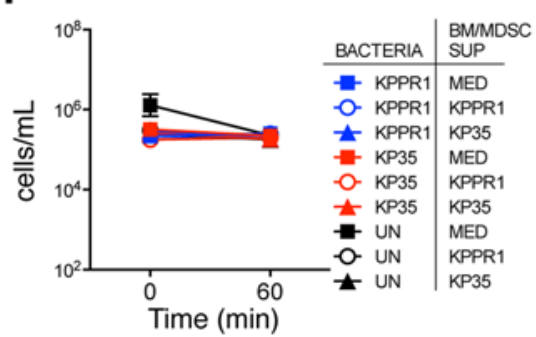

Figure 4. Resistance of KP35 to myeloidderived suppressor cell (MDSC) and neutrophil killing. Bacterial survival of KP35 (red) and KPPR1 (blue) ( $\mathrm{MOI}$ of 1 ) in the presence of: (A) MDSCs derived from bone marrow monocytes (BM/MDSCs), polarized ex vivo $(n=6)$ with (B) MDSC survival over the course of infection, and (C) freshly isolated neutrophils (NEUTs) from BM $(n=4)$ with (D) neutrophil survival. ${ }^{*} P<0.05$ compared with NEUTs with KP35. (E and F) Inhibition of neutrophil killing of KPPR1 or KP35 (MOI of 1) by supernatant (SUP) harvested from BM/MDSCs stimulated with KP35 or KPPR1 (MOI of 10) or a media control (MED), $n=9$. ${ }^{*} P<0.05$ compared with KPPR1 infection with KPPR1 or KP35 infected SUP. There was no change in cell viability over time (B, D, and $\mathbf{F}$ ). Graphs are compiled from 2 (A-D) or 3 independent experiments (E and $\mathbf{F}$ ). For all graphs, each data point is the mean value \pm SEM. ${ }^{*} P<0.05$ by 2 -way ANOVA. For all analyses, Bonferroni's correction for multiple comparisons was performed.

The proteomic data were confirmed with functional assays. The importance of calpain activity in phagocytic killing was illustrated by the effects of the calpain inhibitor calpeptin (CPEP) (32), which increased KPPR1 survival from $8.1 \%$ to $43.6 \%$ and enhanced KP35 proliferation in the presence of viable neutrophils (Figure 5E). We compared the ability of KPPR1 and KP35 to activate $\mathrm{Ca}^{2+}$ fluxes in Fluo-4/ AM-loaded murine neutrophils and found a significant diminution in $\mathrm{Ca}^{2+}$ fluxes evoked by KP35 as compared with KPPR1 (Figure 5F and Supplemental Video 1, A-C). These results suggest that the adaptation of KP35 to the human airway includes mechanisms to suppress the $\mathrm{Ca}^{2+}$-dependent signaling in neutrophils necessary for phagocytic killing. The host responds by increased expression of the many components of the phagocytic process, but with limited efficacy.

Acquisition of unique OGs contribute to KP35 predominance. We sought to identify the genetic basis for the altered behavior of the KP35 isolate by comparing the whole-genome sequence of KP35 with the published genomes of 2 other ST258 K. pneumoniae strains (7) as well as KPPR1 (33) (Figure 6A and Supplemental Table 1). KP35 was closely related to the other ST258 strains but harbored 4 unique OGs including $\operatorname{arcD}$, a component of the arginine deaminase pathway (Supplemental Table 2), which is important in arginine transport. There were 150 OGs shared by both the KP35 and NJST258-1 strains but absent in KPPR1 (Figure 6B). Notably, 26 of these 150 OGs were proteinases, and another 51 OGs consisted of functional proteins related to metabolism, motility, restriction-modification systems, or antimicrobial resistance (Supplemental Table 3). KP35 lacks 18 OGs present in the NJST258-1 strain that include genes potentially involved in stimulating host defenses such as a fimbrial component, an endochitinase, and a component of selenium reductase (Supplemental Table 4). A maximum likelihood (ML) phylogenetic tree illustrates additional divergence of the KP35 core genome, even from recently sequenced ST258 clinical isolates from a geographically contiguous site (i.e., New Jersey) (Figure 6C).

The acquisition of $\operatorname{arcD}$ was of interest since BLASTP analysis indicated that the 332-amino acid $A r c D$ protein is also present in $K$. pneumoniae strains previously associated with clinical outbreaks, including the geographically diverse UHKPC4, UHKPC23, MGH45, and BIDMC 18C strains. Nearly half (32/69, 46\%) of the CRKP within clonal complex 258 from our institution were $\operatorname{arc} D$ positive, also present in isolates from the 2012-13 CRKP cluster at the NIH (34). Functionally, enhanced ability to transport arginine in the setting of an arginine-deficient environment, a consequence of the arginase-producing M-MDSCs, might provide 
A

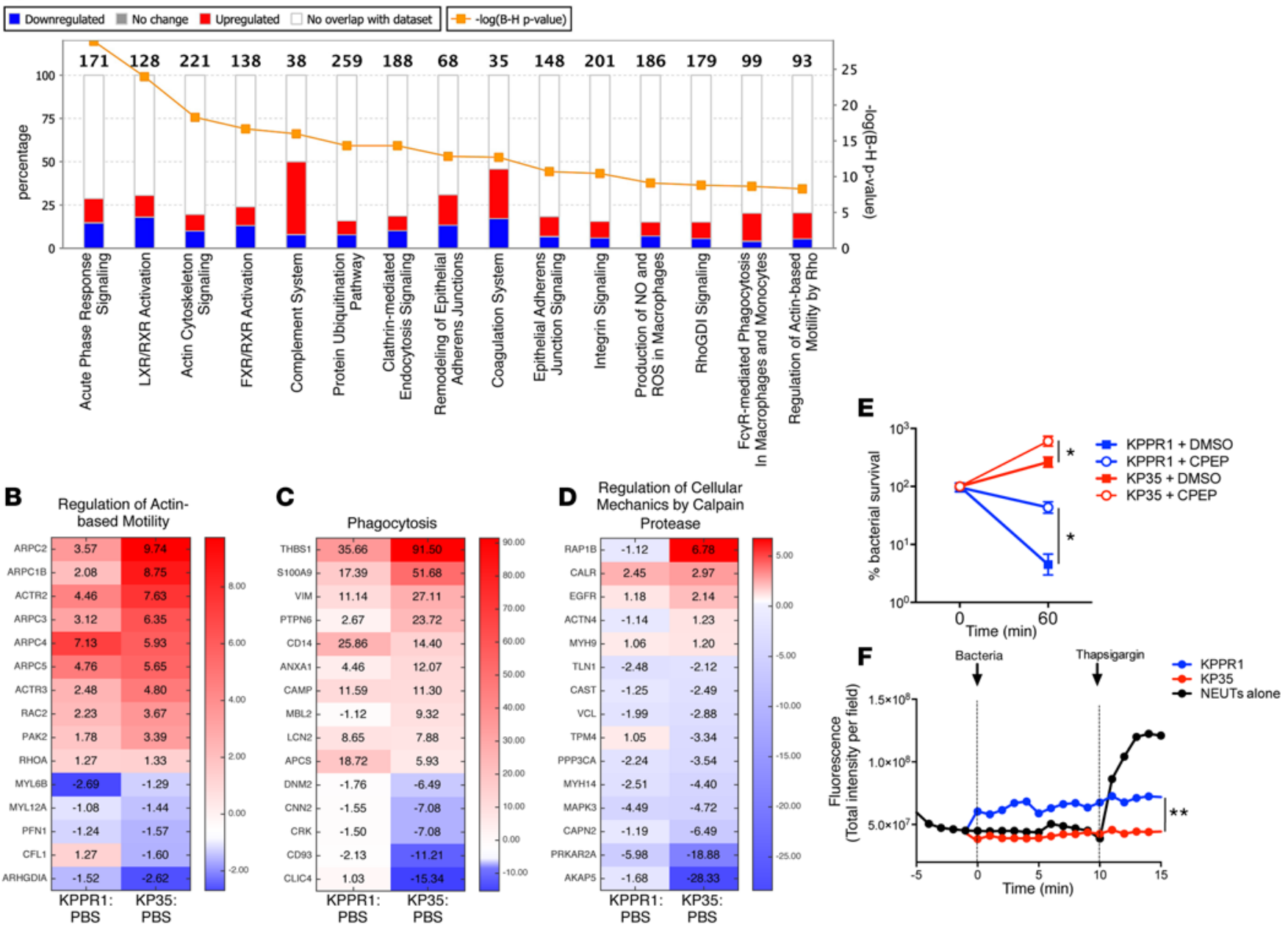

Figure 5. Global changes in host signaling induced by KP35 and KPPR1. (A) The major canonical pathways significantly affected by KP35 are shown in order of statistical significance. Spectral counts for the 1,638 proteins in the pooled bronchoalveolar lavage fluid $(n=3)$ were uploaded into ingenuity pathway analysis software. Numbers above the columns are the number of proteins within each group, with colored bars representing the proportion of upand downregulated genes with KP35 infection as compared with PBS control. Subgroup analysis reflects the differential abundance of specific proteins in KPPR1 and KP35 infection, normalized to PBS controls. This analysis identified (B) actin cytoskeletal remodeling, (C) phagocytosis, and (D) Ca ${ }^{2+} / \mathrm{calpain}$ signaling pathways as differentially affected by KP35 as compared with KPPR1. (E) Functional confirmation of the importance of Ca ${ }^{2+}$ fluxes in phagocytic killing. Percentage of KPPR1 (blue) and KP35 (red) (MOI of 1) killing by neutrophils in the presence of calpeptin (CPEP) compared with DMSO control. ${ }^{*} P$ $<0.05$, Mann-Whitney test, $n=8$. (F) Differential activation of Ca ${ }^{2+}$ fluxes by KP35 and KPPR1. Ca ${ }^{2+}$ fluxes were measured in murine neutrophils (NEUTs) loaded with AM/Fluo-4 prior to stimulation with KP35 and KPPR1 (MOI of 100) or media alone followed by thapsigargin (1 $\mu$ M) as a positive control. Total field fluorescence was measured at each time point using Image). ${ }^{* *} P<0.05$, 2-way ANOVA, Bonferroni's correction for multiple comparisons. Data were compiled from $\mathbf{E}$ or are representative $(\mathbf{F})$ of at least 3 independent experiments.

increased fitness for KP35 and promote its selection from the mixed population found in the lung (35).

To test this hypothesis we identified a ST258 strain, NR1155, closely related to KP35 but lacking arcD, as well as the other 3 potentially new OGs found in KP35. In addition to these 4 OGs, KP35 and NR1155 differ by a number of additional features. These include 352 SNPs (including 77 nonsynonymous and 1 premature stop codon) as well as a larger repertoire of resistance genes in NR1155, which harbors several $\beta$-lactamases (CTX-M-15, OXA-1, OXA-9, and TEM-191), tetracycline resistance-conferring tet $A$ and tet $R$, and KPC-2 rather than KPC-3 in KP35. A competitive index experiment was performed to determine if KP35 has a fitness advantage over NR1155 (Figure 7). Mice were inoculated with the same total inoculum of NR1155, but with a 1:10 ratio of NR1155/KP35 (total inoculum of $10^{8} \mathrm{CFU} /$ mouse) or at a $1: 1$ ratio with a total inoculum of $10^{7} \mathrm{CFU} /$ mouse. Bacterial CFU were quantified over the course of a 4-day infection. Under both conditions, KP35 outcompeted NR1155 in most of the tissues sampled, once beyond the initial infection (Figure 7, A and B). The median proportion of NR1155 in the total inoculum measured at 
A

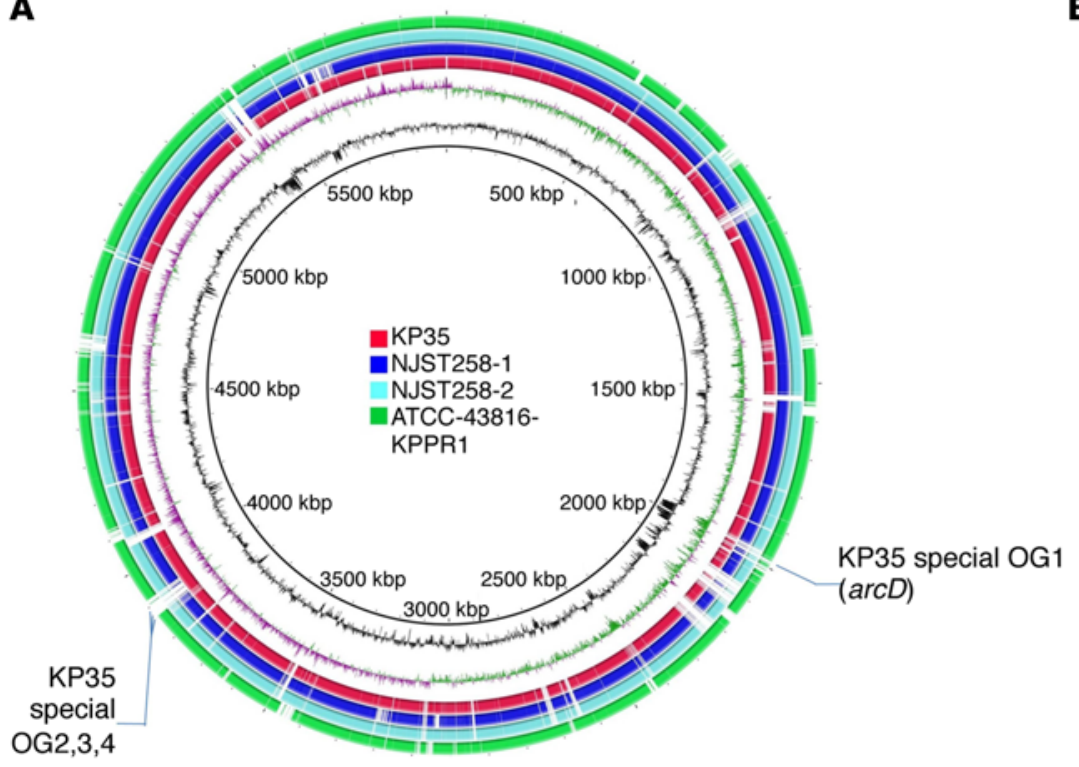

B

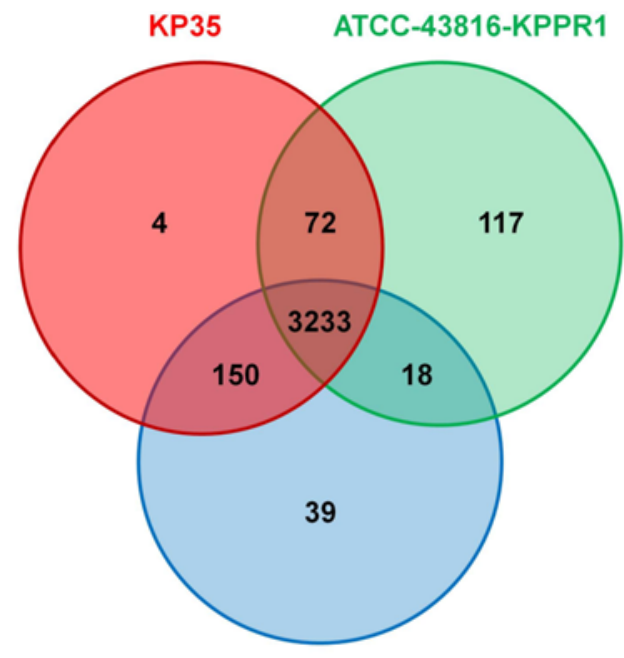

NJST258-1 c

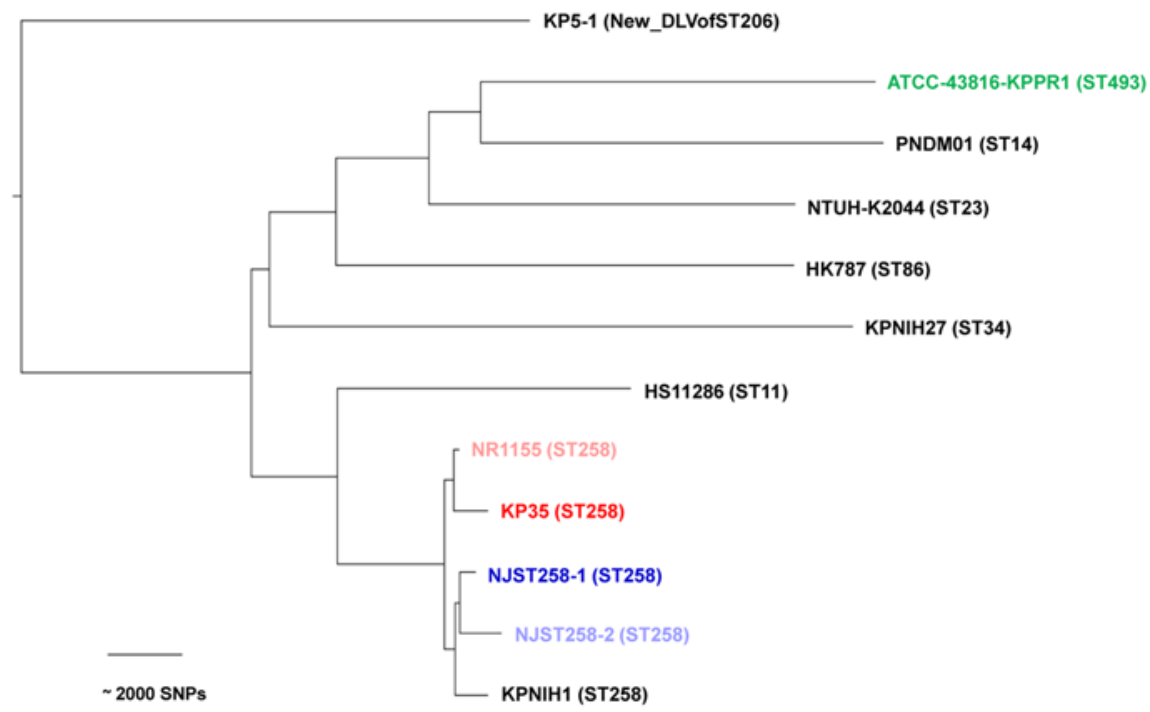

Figure 6. Comparative genomics of KP35, NR1155, published ST258 strains, and the KPPR1 reference strain. (A) Whole-genome alignment of the $4 \mathrm{~K}$. pneumoniae genomes. The de novo-assembled KP35 draft genome and 2 other ST258 reference genomes (NJST258-1 and NJST258-2), aligned to the published ATCC-43816-KPPR1 genome. From the inner to the outer circles: scale bar, GC content, GC skew, KP35, NJST258-1, NJST258-2, ATCC-43816-KPPR1. The location of the KP35 special orthologous groups (OGs), in particular ArcD, were marked. (B) Venn diagram of the proteome OGs, KP35, ATCC-43816-KPPR1, and NJST258-1 showing the number of common and distinct OGs between KP35, ATCC-43816-KPPR1, and NJST258-1. (C) RaxML phylogenetic tree representing genetic origins of ATCC-43816-KPPR1, NR1155, KP35, and multiple clinical isolates of ST258. The tree was based on 79,458 concatenated core genome SNPs of the KP35 and 11 other published $K$. pneumoniae genomes. The multilocus sequence type of each isolate follows in parentheses. Bar represents 2,000 SNPs.

each time point was significantly lower than the baseline (or initial proportion) in all tissues analyzed for both inoculum ratios ( $P<0.01$, using chi-squared test for trend) (Figure 7, C and D). Of note, mice were inoculated with an equivalent amount of NR1155 ( $\left.10^{7} \mathrm{CFU}\right)$ in both conditions, but mice with a lower inoculum of $\mathrm{KP} 35\left(10^{7} \mathrm{CFU}\right)$ lost more weight than those that received the higher dose $\left(10^{8} \mathrm{CFU}\right)$, supporting our observation that KP35 dampens the proinflammatory consequences of infection (Figure 7E). These experiments are consistent with the hypothesis that the acquisition of novel genes by this genetically flexible organism contributes to its fitness and persistence. 
A

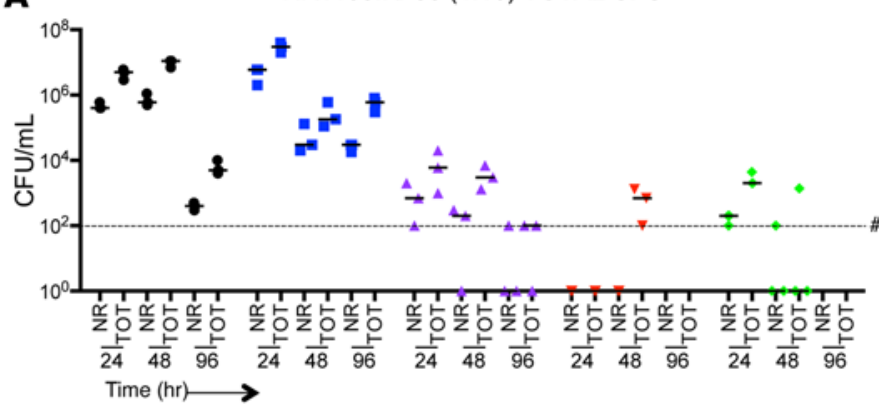

C

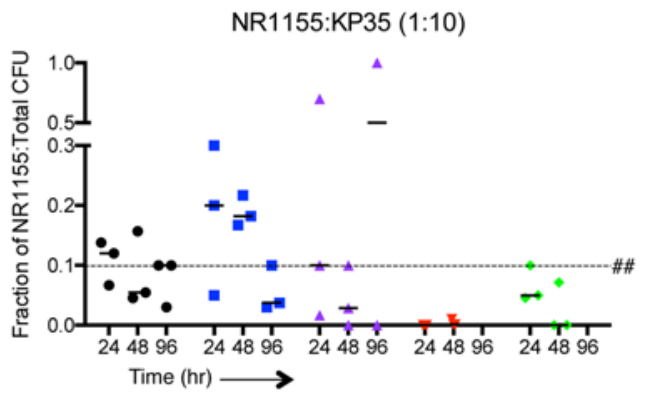

B

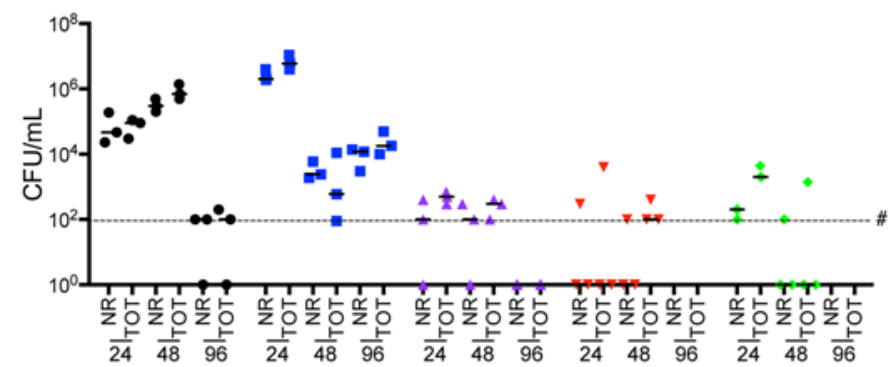

D

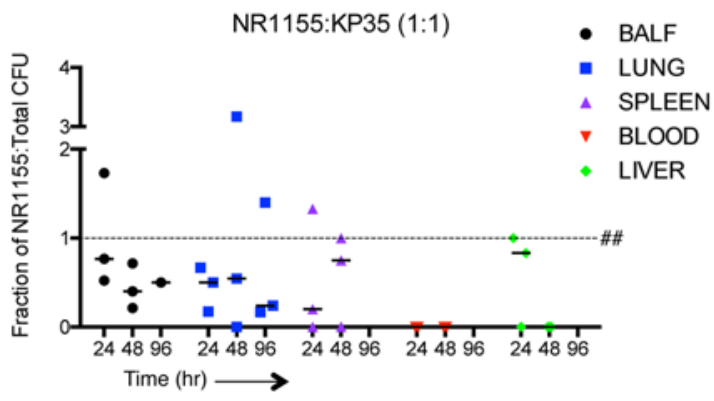

E

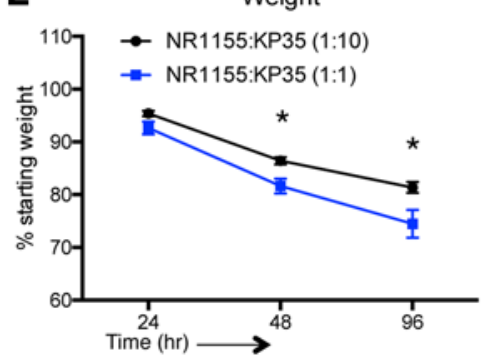

Figure 7. KP35 enjoys a fitness advantage over NR1155. (A and B) A competitive index experiment was performed with NR1155, a closely related isolate to KP35, missing the 4 KP35 special ortholog groups including $\operatorname{arc} D$ but harboring the tetracycline resistance genes tet $A$ and tetR. Mice were inoculated intranasally with $10^{8} \mathrm{CFU}$ at a 1:10 ratio of NR1155/KP35 or $10^{7}$ CFU at a 1:1 ratio and bacterial CFU were quantified. (C and D) Ratio of the tetracycline-resistant isolate NR1155 to the total quantified bacterial load was determined by serial dilution. \#\# = baseline inoculum. Total CFU recovered from different tissues. NR, NR1155 grown on tetracycline-impregnated LB plates; TOT, total CFU enumerated on LB-alone plates. \# = the lower limit of detection. Each data point represents a mouse and the horizontal line is the median. The median proportion of NR1155 in the total inoculum measured at each time point was significantly lower than the baseline (or initial proportion) in all tissues analyzed for both inoculum ratios. $P<0.01$, using chi-squared test for trend. (E) Weight loss of the mice over the course of a 4-day infection. ${ }^{*} P<0.05,2$-way ANOVA with Bonferroni's correction for multiple comparisons

\section{Discussion}

K. pneumoniae is a common cause of serious infection, increasing in prevalence in health care-associated settings (1), and also spreading into the community (36). As a consequence of tremendous genetic flexibility and adaptation to antimicrobial and immune pressures, CRKP has become a major public health problem. The accumulating literature suggest that $K$. pneumoniae, even the ST258 clones, is not a homogeneous group but may differentially express major groups of genes that affect pathogenesis. The relationship between virulence and pathogenicity of bacterial infection is complex, with the majority of the outcome determined by the susceptibility of the host (37). We determined that KP35 was less virulent based on the severely dampened inflammatory response early in infection and the persistence of what we believe to be a high bacterial burden, with $10^{4} \mathrm{CFU} / \mathrm{ml}$ in the BALF at 4 and 7 days of infection. However, this does not mean these opportunists are not pathogenic, as chronic colonization and persistence not only selects for more fit organisms, as we demonstrate, but also increases the potential for invasive infection and further development of antimicrobial resistance, particularly in vulnerable patient populations. The emergence of KP35 as a predominant isolate from among the ST258 strains suggests that ongoing adaptation of $K$. pneumoniae to innate immunity promotes enhanced fitness independently of the presence of antimicrobial selective pressure.

Recent analyses of several $K$. pneumoniae isolates, including ST258 strains, demonstrate the importance of proinflammatory signaling in bacterial clearance. The role of TNF-induced ILC3 production of IL-17A in monocyte-mediated eradication of infection was recently demonstrated (11). IL-17 and TNF were also shown to be critical in the host response to the KPPR1 strains, more virulent strains associated with pathological activation of inflammation $(12,13,38)$ KP35 isolates also cause a brisk proinflammatory response early in infection, as evidenced by the acute cellular and cytokine influx into the infected airway within 
the first 24 hours of infection. However, in contrast with KPPR1 infection, proinflammatory signaling is rapidly suppressed, as evidenced by the attenuation of STAT1 activation, change in cytokine profiles in the BALF, and relatively limited clearance of the infecting organisms.

Our data suggest that this shift in immune signaling is due to the dominance of the immunosuppressive M-MDSCs in the response to KP35, monocytes well known to prevent effective immune clearance of tumors (25). Proteomic data and functional assays further illustrated the profound effects of the M-MDSC population in decreasing phagocytic efficiency without participating in clearance themselves. The importance of monocytes in the clearance of $K$. pneumoniae from the lung has been well recognized $(14,22)$. Exactly why these strains of $K$. pneumoniae preferentially recruit a monocytic response remains unclear. However, once recruited into the airway the autostimulatory effects of these $\mathrm{Ly} 6 \mathrm{C}^{+}$monocytes and their robust production of monocyte chemokines enables them to dominate the milieu of the K. pneumoniaeinfected lung. This, in turn, drives the selection for organisms that can persist alongside them. Unlike inflammatory monocytes that have phagocytic capabilities, our results suggest that the Ly6 ${ }^{\text {hi }}$ population poses no threat to KP35 and that the presence of the immunosuppressive M-MDSCs undoubtedly contributes to KP35 emergence as a pulmonary pathogen.

Whole-genome sequencing indicated the acquisition of several genes during the evolution of the KP35 genome from previously studied ST258 strains. The presence of the arginine transporter $\operatorname{arcD}$ is most consistent with its selection in the setting of the arginase-producing MDSCs. Selective metabolic advantage is provided by $\operatorname{arc} D$ in the evolution of $P$. aeruginosa in the cystic fibrosis lung $(39,40)$ as well as in Streptococcus spp. infections $(41,42)$. To rigorously test the role of $\operatorname{arc} D$ in the pathogenesis of pneumonia, the construction of an $\operatorname{arcD}$ mutant would be optimal. However, the technical challenges of acquired antimicrobial resistance in the ST258 background forced us to compare the fitness of KP35 with the close relative NR1155, which lacks $\operatorname{arcD}$ as well as the 3 other OGs found in KP35. As these genes are not contiguous on the chromosome, it is difficult to predict if all contribute significantly to ST258 adaptation to the lung and to M-MDSCs specifically. Nonetheless, competitive index experiments highlighted the significant advantage that KP35 has gained with the acquisition of 4 unique OGs not found in NR1155, but did not pinpoint $\operatorname{arcD}$ as the sole cause.

Resistance to neutrophil-mediated killing was also an important property of KP35, along with other ST258 isolates, and perhaps is a reason for the abundant monocyte response to infection. Kobayashi et al. suggested that the limited phagocytic killing of ST258 isolates of $K$. pneumoniae was due to impairment in cellular uptake (10), though not eliminating the possibility that neutrophils are preventing bacterial replication or failing to die after infection. We conclude similarly that neutrophils are ineffective in bacterial eradication, though playing some role in bacterial clearance as their selective depletion leads to increased bacterial burden (Supplemental Figure 6C), consistent with previous reports (8). Our data suggest that there are elements of frustrated phagocytosis in the host-pathogen interaction, as markedly increased amounts of cytoskeletal proteins are released into the BALF of infected mice, at a time when the organisms are not being efficiently cleared from the airways. KP35 differs significantly from previously sequenced strains that readily activate $\mathrm{Ca}^{2+}$ signaling, required for calpain activation and phagocytic uptake. KP35 completely failed to induce $\mathrm{Ca}^{2+}$ fluxes in immune cells, and numerous $\mathrm{Ca}^{2+}$-dependent substrates such as the S100A9 proteins and thrombospondin were significantly upregulated in KP35 infection. Although the mechanism underlying this disruption of $\mathrm{Ca}^{2+}$ signaling remains to be established, this adaptation clearly facilitates avoidance of host immune cell clearance.

This analysis of a locally predominant clone of the globally disseminated $K$. pneumoniae ST258 highlights the tremendous adaptability of this pathogen and the challenges in designing effective microbial eradication strategies. In contrast with some airway pathogens that reliably activate an excessive proinflammatory response in the setting of acute infection (43), it would be difficult to predict what host signaling will be activated by any given K. pneumoniae isolate. As suggested in Figure 1, even from a single patient, there is ongoing bacterial adaptation readily detected through the changing patterns of antimicrobial resistance and likely paralleled with the acquisition of phenotypically silent genes that thwart innate immune clearance. The difficulty in predicting the overall immune milieu within the infected airway is similar to that observed in sepsis, in which it is often critical but impossible to establish if there is too much, too little, or sufficient inflammation to effectively eradicate infection (44).

At least for the KP35 ST258 strain, our results suggest that the failure of the host to eradicate this organism is due to the early predominance of immunosuppressive M-MDSCs that terminate the proinflammatory signaling required for $K$. pneumoniae clearance and the dynamic adaptation of the infecting organisms to 
those cells. A comprehensive analysis of the unique characteristics of the evoked immune response to a specific pathogen and genomic analysis of the pathogen itself could become important tools in defining mechanisms that promote persistence of such adaptable pathogens within the lung.

\section{Methods}

\section{Study isolates and chart review}

We selected the representative clinical isolate KP35 from a patient with bacteremia for comprehensive study (1). It was chosen because it is a common isolate recovered in our institution and it expresses the most common cps and wzi genotypes. Antibiogram data were assembled from a retrospective chart review of a representative patient, under protocol AAAL1023, approved by the Institutional Review Board of the Columbia University Medical Center. Antibiotic susceptibility testing was performed according to routine microbiology laboratory protocols using the Vitek 2 automated system, with additional E-testing as needed (bioMérieux). Susceptibility breakpoints were derived from Clinical and Laboratory Standards Institute guidelines (45). Clinical isolate NR1155 was selected from our repository after whole-genome sequencing owing to its lack of the 4 unique OGs of KP35 and its resistance to tetracycline.

\section{Cell lines and bacterial strains}

Immortalized BMDMs (WT, Tlr4 ${ }^{-/}$, and Trif ${ }^{\prime-}$ ) (Generously provided by K. Fitzgerald at the University of Massachusetts Medical School, Worcester, Massachusetts, USA) were grown at $37^{\circ} \mathrm{C}$ with $5 \% \mathrm{CO}_{2}$ in RPMI (Corning) with 10\% heat-inactivated FBS (Gibco). Purified E. coli LPS (Sigma-Aldrich) was used at the indicated concentrations. KP35 and NR1155 as well as K. pneumoniae ATCC 43816 (KPPR1) and $P$. aeruginosa (PAK) were grown in LB and resuspended in PBS for in vivo infections or respective cell culture media for in vitro assays. The concentration of bacteria for any given assay was enumerated after serial dilution and plating on enriched agar. To quantify cell culture supernatant protein levels, a mouse TNF ELISA was used (Biolegend).

\section{Mouse studies}

In vivo experiments were performed using 8-week-old, male C57BL/6J mice (Jackson Laboratories). Mice were anesthetized with $100 \mathrm{mg} / \mathrm{kg}$ ketamine and $5 \mathrm{mg} / \mathrm{kg}$ xylazine given i.p. and infected intranasally with KP35 (10 ${ }^{8} \mathrm{CFU}$ in $50 \mu \mathrm{l}$ of PBS). For the competitive index experiment, mice were inoculated intranasally with $10^{8} \mathrm{CFU}$ at a $1: 10$ ratio of NR1155/KP35 or $10^{7} \mathrm{CFU}$ at a $1: 1$ ratio. Bacterial load of each compartment was quantified by serial dilutions on enriched agar. Animal experiments were performed in accordance with the guidelines of the IACUC at Columbia University (protocol number AAAG9307) or the Bioethics and Biosafety Committee of the Pontificia Universidad Católica de Chile (CEC 150721005).

\section{Histopathology and whole-lung imaging}

Whole mouse lung was fixed with $4 \%$ paraformaldehyde for 24 hours, $70 \%$ ethanol for 24 hours, and then prepared in paraffin blocks. H\&E or trichrome staining was performed on 5-mm sections for gross pathology. Euthanized mice were imaged using a Quantum FX system, a stand-alone micro CT at the Small Animal Imaging Shared Resource of the Herbert Irving Comprehensive Cancer Center at Columbia University. Image collection, processing, and analysis for this work were performed at this shared resource.

\section{BALF assays}

BALF was obtained by instilling aliquots of sterile PBS with calcium and magnesium into a cannulated trachea. Serial dilutions for bacterial enumeration were performed on the BALF prior to centrifuging. The supernatant of the first $\mathrm{ml}$ of BALF was set aside for cytokine array, a 31-plex mouse discovery assay from Eve Technologies. For comparison of KP35 and PAK infection, cytokines were measure from pooled BALF using a semiquantitative cytokine array (R\&D Systems). The total cellular content was set aside for flow cytometry (FC).

\section{Analysis of immune cell populations}

To further delineate immune cell populations, analysis of cell populations in BALF or single-cell suspensions of lung homogenate was conducted using multicolor FC on a BD LSR II. Cells were labeled with a combi- 
nation of PerCP-Cy5.5-labelled anti-CD11c (N418; Biolegend), fluorescein (FITC)-labeled anti-MARCO (MCA1849FT; Bio-Rad), PE-Cy7-labeled anti-F4/80 (BM8; Biolegend), phycoerythrin (PE)-CF594labeled anti-Ly6C (AL-21; BD Biosciences), PE-labeled anti-CD200R (PK136; eBioscience), BV421-labeled anti-CD86 (GL-1; Biolegend), BV510-labeled anti-CD11b (M1/70; Biolegend), BV605-labeled anti-Ly6G (1A8; BD Biosciences), BV650-labeled anti-CD206/MMR (C068C2; Biolegend), APC-Cy7-labeled antiMHCII (M5/114.15.2, Biolegend), AF700-labeled anti-CD45 (30-F11; Biolegend), and AF647-labeled anti-Siglec F (E50-2440; BD Biosciences). LIVE/DEAD Fixable Dead Cell Stain Kits, blue fluorescent dye (Molecular Probes), and Fc block (anti-mouse CD16/32) (93; Biolegend) was added to each sample.

Uniform dyed microspheres (Bangs Laboratories) were added to calculate the concentration of cellular components. All FC data were analyzed on FlowJo (ver 10.0.8). Research reported in this publication was performed in the CCTI Flow Cytometry Core, supported in part by the Office of the Director, National Institutes of Health. The content is solely the responsibility of the authors and does not necessarily represent the official views of the National Institutes of Health.

Ly $6 \mathrm{C}^{+}$cell population isolation and qRT-PCR

WT mice were infected intranasally with $10^{8} \mathrm{CFU}$ of KP35 in $50 \mu 1$ of PBS, $50 \mu \mathrm{g}$ E. coli LPS ( $\left.1 \mathrm{mg} / \mathrm{ml}\right)$, or 50 $\mu \mathrm{PBS}$ for 48 hours. BM cells from 1 femur were flushed with $1 \mathrm{ml}$ of PBS and cells from BALF (as described above) were isolated from these mice. After RBC lysis, cells were incubated with Ly6 $\mathrm{C}^{+}-\mathrm{PE}$ followed by PEmagnetic beads and isolated through positive selection (Miltenyi Biotec). Isolation purity was confirmed with FC (Supplemental Figure 5A). RNA was first isolated from these cells using an E.Z.N.A Total RNA Kit I (Omega bio-tek), transcribed to cDNA using a High Capacity cDNA Reverse Transcription Kit (Applied Biosystems), and finally qRT-PCR was performed using relevant primers and Power SYBR Green PCR Master Mix (Applied Biosystems) on a StepOnePlus Real-time PCR System (Applied Biosystems), using StepOne Software (Ver 2.2.2). The following target genes and their primers are listed here: $C c l 2$ (Fwd - CAGGTCCCTGTCATGCTTCT, Rev - TCTGGACCCATTCCTTCTTG); Il10 (Fwd - GCTCTTACTGACTGGCATGAG, Rev - CGCAGCTCTAGGAGCATGTG); Arginase1 (Fwd - CGCCTTTCTCAAAAGGACAG, Rev - ACAGACCGTGGGTTCTTCAC); Retnla (Fzz-1) (Fwd - CTCCACTGTAACGAAGACTC, Rev - GCAGTGGTCCAGTCAACGA); Inos (Fwd - TCCTCACTGGGACAGCACAGAATG, Rev - GTGTCATGCAAAATCTCTCCACTGCC); Socs3 (Fwd - GGGTGGCAAAGAAAAGGAG, Rev - GTTGAGCGTCAAGACCCAGT); Kc/Cxcl1 (Fwd - CCGCGCCTATCGCCAATGAGCTGCGC, Rev - CTTGGGGACACCTTTTAGCATCTTTTGG); Tnf (Fwd - ATGAGCACAGAAAGCATGATC, Rev - TACAGGCTTGTCACTCGAATT); and Actin (Fwd - CCTTTGAAAAGAAATTTGTCC, Rev AGAAACCAGAACTGAAACTGG).

\section{BM/MDSC differentiation and infection}

$\mathrm{BM} / \mathrm{MDSCs}$ were differentiated as previously described (46), with some modifications. Briefly, $5 \times 10^{6}$ BM cells were seeded into 100-mm dishes in $10 \mathrm{ml}$ of RPMI 1640 containing $2 \mathrm{mM}$ L-glutamine, 10 $\mathrm{mM}$ HEPES, $20 \mu \mathrm{M}$ 2-ME, 1\% streptomycin/penicillin, and 10\% heat-inactivated FBS supplemented with GM-CSF (40 ng/ml) and G-CSF (40 ng/ml) (Peprotech). Cells were maintained at $37^{\circ} \mathrm{C}$ and $5 \% \mathrm{CO}_{2}$ for 5 days. On day 5, cells were washed twice and resuspended in RPMI 1640 plus 10\% FBS. To evaluate the bactericidal capacity of the BM/MDSCs, $1 \times 10^{5}$ cells were seeded into 96-well plates and stimulated with $1 \times 10^{5} \mathrm{CFU}$ (MOI of 1 ) of opsonized KPPR 1 or KP35 for $0,1,2$, and 4 hours at $37^{\circ} \mathrm{C}$ with $5 \% \mathrm{CO}_{2}$. Cell viability was measured by counting total cell number in a conventional hemocytometer with trypan exclusion, whereas bacterial viability was measured by serial dilutions plated after cell lysis with $1 \%$ saponin.

\section{Neutrophil isolation and bacterial killing assay}

Neutrophils were isolated from BM cells by density gradient centrifugation as previously described (47). Briefly, BM cells were overlaid on a Histopaque 1119 and Histopaque 1077 gradient and centrifuged for 30 minutes at $720 \mathrm{~g}$ and at room temperature without brake; neutrophils were collected at the interface between the 2 layers, counted, and resuspended in HBSS containing $\mathrm{Ca}^{2+}$ and $\mathrm{Mg}^{2+}$ and supplemented with $0.1 \%$ gelatin. To evaluate bacterial killing capacity, neutrophils were incubated with opsonized KPPR1 or $\mathrm{KP} 35$ (MOI of 1 ) for $0,30,60,90$, and 120 minutes with slow rotation at $37^{\circ} \mathrm{C}$. After each time point, the reaction was stopped at $4^{\circ} \mathrm{C}$ and the total viable cells were counted in a conventional hemocytometer with trypan exclusion. Bacterial viability was determined by serial dilutions after lysing the cells with $1 \%$ 
saponin. To evaluate the role of $\mathrm{Ca}^{2+}$ flux and calpains in the bacterial killing capacity of neutrophils, cells were pretreated with calpeptin $(200 \mu \mathrm{M}) 60$ minutes prior to infection and bacterial killing capacity was measured after 60 minutes. To evaluate the effect of MDSCs on bacterial killing by neutrophils, filtered $(0.2 \mu \mathrm{m})$ supernatant of BM/MDSCs untreated or stimulated with KPPR1 or KP35 (MOI of 10) was added to the media at a 1:1 ratio 60 minutes prior to infection and bacterial killing capacity was measured.

\section{Calcium imaging}

BM-isolated neutrophils were loaded with AM/Fluo-4 $(1 \mu \mathrm{M})$ (Invitrogen) with PowerLoad concentrate $(1 \mu \mathrm{M})$ (Invitrogen) prior to imaging and stimulation. Thapsigargin $(10 \mu \mathrm{M})$ was added to cell culture media as a positive control. Cells were imaged on an Olympus 1X81 inverted microscope with a GFP mercury laser. Videos were made using MetaMorph software (Ver.7.5.3.0, April, 2008) and images were analyzed using ImageJ software (NIH).

\section{Genome sequencing, assembly, and annotation}

Whole-genome sequencing was performed on KP35 (1). DNA was extracted using a QIAamp DNA Blood Mini Kit (QIAGEN) following the manufacturer's instructions. Whole-genome sequencing was performed using the HiSeq 2000 sequencer (Illumina). Unique index-tagged libraries were created in order to generate 125-bp paired-end reads. The de novo assembly of the sequencing reads was performed using the Newbler assembly software. The KP35 chromosome was approximately $5.21 \mathrm{Mb}$ in size with a GC ratio of $57.30 \%$. A total of $377,493,250 \mathrm{bp}$ in 301,9946 reads were obtained and then de novo assembled into 123 large contigs. The genome sequencing and contig details are shown in Supplemental Table 1. A total of 4,984 coding DNA sequences, 70 tRNA-encoding genes, and 1 rRNA locus were detected. Fifty-eight percent (58\%) of the genes were assigned to specific subsystem categories by NMPDR RAST.

\section{Comparative genomic analyses and gene ortholog analysis}

Predicted genes and their translated protein sequences of KP35 (accession \#LRXK000000000) were compared with the reference genome ATCC 43816 KPPR1, NJST258-1, and NJST258-2 (7, 33), and clustered into OGs by using the OrthoMCL software (48). The protein sequences of the 2 reference genomes were calculated based on the NCBI annotations. All versus all BLASTP was performed with the default parameter set (an E-value cutoff of $1 \times 10^{-5}$, a percent match cutoff of $50 \%$, and an inflation value of 1.5). Common and unique OGs identified among the genomes were analyzed by using a Venn diagram (49). Whole-genome alignment was performed by using Mauve (version. 2.3.1) (50), BLAST Ring Image Generator program (BRIG) (51), and Geneious (Biomatters Ltd., New Zealand). Maximum likelihood phylogenetic tree, based on concatenate core genome SNPs, was performed using RAxML BlackBox (52). The full genome of NR1155 is assigned accession number SRP082410.

\section{Shotgun proteomics analysis}

Mice. C57BL/6J mice were given KPPR1 (105 CFU), KP35 (10 8 CFU), or PBS and BALF was harvested 48 hours after inoculation. Equivalent inocula were recovered $\left(10^{6} \mathrm{CFU}\right)$ at this time point despite disparate initial inocula. Proteomic analysis was performed on BALF pooled from 3 mice per experimental condition.

Materials. HPLC-grade buffers, dithiothreitol, acetonitrile ( $\mathrm{ACN})$, ammonium bicarbonate, trifluoroacetic acid (TFA), and iodoacetamide were purchased from Thermo Fisher Scientific. Trypsin Gold, mass spectrometry grade, was purchased from Promega. Nanopure water was prepared with use of Milli-Q water purification system (Millipore).

\section{In-solution protein digestion}

Proteins were precipitated from $500 \mu \mathrm{l}$ of BALF using methanol/chloroform and protein concentrations were determined by the Qubit assay according to the manufacture's instructions (Life Technologies). Proteins $(50 \mu \mathrm{g})$ were reduced, alkylated, and digested with $500 \mathrm{ng}$ Trypsin Gold in $200 \mu 1$ of 50 $\mathrm{mM}$ ammonium bicarbonate at $37^{\circ} \mathrm{C}$ for 16 hours. After digestion, the peptide mix was centrifuged subsequently for 30 minutes at $16,873 \mathrm{~g}$, and the cleared supernatants were transferred to fresh tubes to be dried and resuspended in $0.1 \%$ TFA for subsequent peptide fractionation using the Pierce High $\mathrm{pH}$ Reversed-Phase Peptide Fractionation Kit (Thermo Fisher Scientific). Seven peptide fractions were collected and lyophilized for liquid chromatography/tandem mass spectrometry (LC-MS/MS) analysis. 


\section{Sample preparation}

Proteins from pooled samples of mouse BALF (total volume $500 \mu \mathrm{l}$ ) were precipitated by methanol/chloroform and resuspended in $20 \mu \mathrm{l}$ of $4 \mathrm{M}$ urea in $50 \mathrm{mM}$ ammonium bicarbonate. Protein concentration in the mouse lavage was determined by the EZQ Protein Quantification Assay (Life Technologies). BALF proteins $(2 \mu \mathrm{g})$ were digested with $150 \mathrm{ng}$ of trypsin (1:40) along with $2 \mathrm{mM} \mathrm{CaCl}_{2}$ and incubated at $37^{\circ} \mathrm{C}$ for 16 hours. Samples were centrifuged for 30 minutes at $16,873 \mathrm{~g}$, and the cleared supernatants were transferred to fresh tubes to be acidified with $90 \%$ formic acid ( $2 \%$ final) to stop proteolysis. The soluble peptide mixtures were collected for LC-MS/MS analysis.

\section{LC-MS/MS analysis}

The concentrated peptide mix was reconstituted in a solution of $2 \% \mathrm{ACN}, 2 \%$ formic acid (FA) for MS analysis. Peptides were loaded with the autosampler directly onto a 2-cm C18 PepMap precolumn and were eluted from the $50 \mathrm{~cm} \times 75 \mu \mathrm{m}$ ID PepMap RSLC C18, 2- $\mu \mathrm{m}$ column using a Thermo Dionex 3000 with a 98 -minute gradient from $2 \%$ buffer $\mathrm{B}$ to $30 \%$ buffer $\mathrm{B}(100 \% \mathrm{ACN}, 0.1 \% \mathrm{FA})$. The gradient was switched from $30 \%$ to $85 \%$ buffer B over 5 minutes and held constant for 1 minute. Finally, the gradient was changed from $85 \%$ buffer B to $98 \%$ buffer A (100\% water, $0.1 \%$ FA) over 2 minutes, and then held constant at $98 \%$ buffer A for 25 more minutes. The application of a $2.0 \mathrm{kV}$ distal voltage electrosprayed the eluting peptides directly into the mass spectrometer equipped with an EASY-Spray source (Thermo Finnigan). Full mass spectra were recorded on the peptides over a 400 to $1500 \mathrm{~m} / z$ range at 120,000 resolution, followed by MS/MS CID (collision induced dissociation) events for a total of a 3-second cycle. Charge state-dependent screening was turned off, and peptides with a charge state of 2 to 6 were analyzed. MS-scanning functions and HPLC gradients were controlled by the Xcalibur data system (Thermo Finnigan). Three technical replicates were run for each sample, and MS/MS data from technical replicates were merged for subsequent database searches.

\section{Database searches and interpretation of MS/MS data}

MS/MS spectra from raw files were searched against a human protein database using the Proteome Discoverer 1.4 (Thermo Finnigan). The Proteome Discoverer application extracts relevant MS/MS spectra from the .raw file and determines the precursor charge state and the quality of the fragmentation spectrum. The Proteome Discoverer probability-based scoring system rates the relevance of the best matches found by the SEQUEST algorithm (53). The mouse database was downloaded as FASTA-formatted sequences from Uniprot protein database (database released on December, 2014). The peptide mass search tolerance was set to $10 \mathrm{ppm}$. A minimum sequence length of 7 amino acids was required. Only fully tryptic peptides were considered. To calculate confidence levels and FDR, Proteome Discoverer generates a decoy database containing reverse sequences of the non-decoy protein database and performs the search against this concatenated database (non-decoy + decoy) (54). The discriminant score was set at $1 \%$ FDR, which was determined based on the number of accepted decoy database peptides to generate protein lists for this study. Spectral counts used to identify each protein were used for expression profiling analysis. Qlucore Omics Explorer (Qlucore AB) was used to perform statistical analysis of quantifiable proteins among biological replicates ( $t$ test, $P<0.05$ ). Differentially expressed proteins were analyzed using DAVID (55).

\section{Ingenuity network analysis}

We applied network analysis to a selected protein list from global proteomics expression profiling data using the ingenuity pathway analysis (IPA) algorithm (QIAGEN). Proteins whose expression was changed by heterogeneous nuclear ribonucleoprotein A/B disruption were uploaded into IPA (http://www.ingenuity.com) to examine functional relationships among these proteins based on a database created from previously published peer-reviewed papers found in NCBI PubMed, Medline, and several other databases. Then, IPA associates these selected proteins with biological pathways using the Ingenuity Knowledge Base. Right-tailed Fisher's exact test with the Benjamini-Hochberg multiple correction was used to control for false positives and calculate a $P$ value determining the probability that each biological function and/or disease assigned to that data set is due to chance alone.

\section{Statistics}

All statistical analysis was performed using GraphPad Prism Version 6.0c (March 21, 2013). For most data sets, either a 1-way or 2-way ANOVA was performed with posttest analysis for multiple comparisons as 
described in the congruent figure legend. For data sets in which only 2 data sets could be compared, a 2-tailed Mann-Whitney test was performed. A $P$ value of $<0.05$ was considered significant. For the competitive index experiment, a chi-squared test for trend was used to compare recovered CFU, with $P<0.01$ considered significant. Grubb's test was performed on all data sets to remove significant outliers with an $\alpha$ value of 0.05 .

\section{Author contributions}

DA, ACU, and ASP designed experiments, analyzed data, and wrote the manuscript. DA, HP, and MW designed, performed and analyzed experiments. ZW and ACU performed and analyzed the comparative bacterial genomics. PP, AK, and EIC performed the proteomics.

\section{Acknowledgments}

This work was supported by NIH RO1 HL073989 (to AP), NIH RO1 AI116939 01 (to AU), NIH K12HD047349-08 (to DA), and FONDECYT 1140010 and Millennium Institute on Immunology and Immunotherapy P09/016-F (to HP and SB). The proteomics core facility is funded by NIH P30 CA01369639S3, CCTI Flow Core by NIH S10 RR027050, and the Small Animal Imaging Shared Resource Small by NIH P30 CA013696. The authors declare no competing financial interests. Fluorescence microscopy was performed in the laboratory of Jahar Bhattacharya with the assistance of Rebecca Hough and Mohammed Naeem Islam at the Columbia University Medical Center. Statistical analysis was guided by Philip Zachariah at the Columbia University Medical Center.

Address correspondence to: Alice Prince, 650 W 168th Street, Black Building Room 416, New York, New York 10032, USA. Phone: 212.305.4193; E-mail: asp7@columbia.edu.

1. Gomez-Simmonds A, et al. Population structure of Klebsiella pneumoniae causing bloodstream infections at a New York City tertiary care hospital: diversification of multidrug-resistant isolates. J Clin Microbiol. 2015;53(7):2060-2067.

2. Tumbarello M, et al. Infections caused by KPC-producing Klebsiella pneumoniae: differences in therapy and mortality in a multicentre study. J Antimicrob Chemother. 2015;70(7):2133-2143.

3. Lee GC, Burgess DS. Treatment of Klebsiella pneumoniae carbapenemase (KPC) infections: a review of published case series and case reports. Ann Clin Microbiol Antimicrob. 2012;11:32.

4. Borer A, et al. Attributable mortality rate for carbapenem-resistant Klebsiella pneumoniae bacteremia. Infect Control Hosp Epidemiol. 2009;30(10):972-976

5. Kitchel B, et al. Molecular epidemiology of KPC-producing Klebsiella pneumoniae isolates in the United States: clonal expansion of multilocus sequence type 258. Antimicrob Agents Chemother. 2009;53(8):3365-3370.

6. Diago-Navarro E, et al. Carbapenem-resistant Klebsiella pneumoniae exhibit variability in capsular polysaccharide and capsule associated virulence traits. J Infect Dis. 2014;210(5):803-813.

7. Deleo FR, et al. Molecular dissection of the evolution of carbapenem-resistant multilocus sequence type 258 Klebsiella pneumoniae. Proc Natl Acad Sci U S A. 2014;111(13):4988-4993.

8. Xiong H, et al. Distinct contributions of neutrophils and CCR2 ${ }^{+}$monocytes to pulmonary clearance of different Klebsiella pneumoniae strains. Infect Immun. 2015;83(9):3418-3427.

9. Fodah RA, et al. Correlation of Klebsiella pneumoniae comparative genetic analyses with virulence profiles in a murine respiratory disease model. PLoS One. 2014;9(9):e107394.

10. Kobayashi SD, et al. Phagocytosis and killing of carbapenem-resistant ST258 Klebsiella pneumoniae by human neutrophils. J Infect Dis. 2016;213(10):1615-1622.

11. Xiong H, Keith JW, Samilo DW, Carter RA, Leiner IM, Pamer EG. Innate lymphocyte/Ly6C(hi) monocyte crosstalk promotes Klebsiella pneumoniae clearance. Cell. 2016;165(3):679-689.

12. Zeng X, Moore TA, Newstead MW, Hernandez-Alcoceba R, Tsai WC, Standiford TJ. Intrapulmonary expression of macrophage inflammatory protein 1alpha (CCL3) induces neutrophil and NK cell accumulation and stimulates innate immunity in murine bacterial pneumonia. Infect Immun. 2003;71(3):1306-1315.

13. Ye P, et al. Interleukin-17 and lung host defense against Klebsiella pneumoniae infection. Am J Respir Cell Mol Biol. 2001;25(3):335-340.

14. Herold S, et al. Exudate macrophages attenuate lung injury by the release of IL-1 receptor antagonist in gram-negative pneumonia. Am J Respir Crit Care Med. 2011;183(10):1380-1390.

15. Marvel D, Gabrilovich DI. Myeloid-derived suppressor cells in the tumor microenvironment: expect the unexpected. JClin Invest. 2015;125(9):3356-3364.

16. Bronte V, et al. Recommendations for myeloid-derived suppressor cell nomenclature and characterization standards. Nat Commun 2016;7:12150.

17. Tzouvelekis LS, et al. KPC-producing, multidrug-resistant Klebsiella pneumoniae sequence type 258 as a typical opportunistic pathogen. Antimicrob Agents Chemother. 2013;57(10):5144-5146.

18. Lawlor MS, Hsu J, Rick PD, Miller VL. Identification of Klebsiella pneumoniae virulence determinants using an intranasal infection model. Mol Microbiol. 2005;58(4):1054-1073.

19. Moore TA, Perry ML, Getsoian AG, Newstead MW, Standiford TJ. Divergent role of gamma interferon in a murine model of 
pulmonary versus systemic Klebsiella pneumoniae infection. Infect Immun. 2002;70(11):6310-6318.

20. Happel KI, et al. Divergent roles of IL-23 and IL-12 in host defense against Klebsiella pneumoniae. J Exp Med. 2005;202(6):761-769.

21. Broug-Holub E, et al. Alveolar macrophages are required for protective pulmonary defenses in murine Klebsiella pneumonia: elimination of alveolar macrophages increases neutrophil recruitment but decreases bacterial clearance and survival. Infect Immun. 1997;65(4):1139-1146.

22. Poe SL, et al. STAT1-regulated lung MDSC-like cells produce IL-10 and efferocytose apoptotic neutrophils with relevance in resolution of bacterial pneumonia. Mucosal Immunol. 2013;6(1):189-199.

23. Najjar I, Fagard R. STAT1 and pathogens, not a friendly relationship. Biochimie. 2010;92(5):425-444.

24. Condamine T, Mastio J, Gabrilovich DI. Transcriptional regulation of myeloid-derived suppressor cells. J Leukoc Biol. 2015;98(6):913-922.

25. Gabrilovich DI, Nagaraj S. Myeloid-derived suppressor cells as regulators of the immune system. Nat Rev Immunol. 2009;9(3):162-174.

26. Hu X, et al. Transmembrane TNF- $\alpha$ promotes suppressive activities of myeloid-derived suppressor cells via TNFR2. J Immunol. 2014;192(3):1320-1331.

27. Schumak B, et al. Specific depletion of Ly6C(hi) inflammatory monocytes prevents immunopathology in experimental cerebral malaria. PLoS One. 2015;10(4):e0124080.

28. Vog1 T, et al. MRP8 and MRP14 control microtubule reorganization during transendothelial migration of phagocytes. Blood. 2004;104(13):4260-4268.

29. Srikrishna G. S100A8 and S100A9: new insights into their roles in malignancy. J Innate Immun. 2012;4(1):31-40.

30. Zhao Y, et al. Thrombospondin-1 restrains neutrophil granule serine protease function and regulates the innate immune response during Klebsiella pneumoniae infection. Mucosal Immunol. 2015;8(4):896-905.

31. Adams JC, Lawler J. The thrombospondins. Cold Spring Harb Perspect Biol. 2011;3(10):a009712.

32. Jeong SY, Martchenko M, Cohen SN. Calpain-dependent cytoskeletal rearrangement exploited for anthrax toxin endocytosis. Proc Natl Acad Sci U S A. 2013;110(42):E4007-E4015.

33. Broberg CA, Wu W, Cavalcoli JD, Miller VL, Bachman MA. Complete genome sequence of Klebsiella pneumoniae strain ATCC 43816 KPPR1, a rifampin-resistant mutant commonly used in animal, genetic, and molecular biology studies. Genome Announc. 2014;2(5).

34. Conlan S, Thomas PJ, Deming C, et al. Single-molecule sequencing to track plasmid diversity of hospital-associated carbapenemase-producing Enterobacteriaceae. Sci Transl Med. 2014;6(254):254ra126.

35. Maghnouj A, de Sousa Cabral TF, Stalon V, Vander Wauven C. The arcABDC gene cluster, encoding the arginine deiminase pathway of Bacillus licheniformis, and its activation by the arginine repressor argR. J Bacteriol. 1998;180(24):6468-6475.

36. Stoesser N, et al. Colonization with Enterobacteriaceae producing ESBLs in children attending pre-school childcare facilities in the Lao People's Democratic Republic. J Antimicrob Chemother. 2015;70(6):1893-1897.

37. Casadevall A, Pirofski L. Host-pathogen interactions: the attributes of virulence. J Infect Dis. 2001;184(3):337-344

38. Aujla SJ, et al. IL-22 mediates mucosal host defense against Gram-negative bacterial pneumonia. Nat Med. 2008;14(3):275-281.

39. Hogardt M, Heesemann J. Microevolution of Pseudomonas aeruginosa to a chronic pathogen of the cystic fibrosis lung. Curr Top Microbiol Immunol. 2013;358:91-118.

40. Benkert B, Quäck N, Schreiber K, Jaensch L, Jahn D, Schobert M. Nitrate-responsive NarX-NarL represses argininemediated induction of the Pseudomonas aeruginosa arginine fermentation arcDABC operon. Microbiology (Reading, Engl) 2008;154(Pt 10):3053-3060.

41. Schulz C, Gierok P, Petruschka L, Lalk M, Mäder U, Hammerschmidt S. Regulation of the arginine deiminase system by ArgR2 interferes with arginine metabolism and fitness of Streptococcus pneumoniae. MBio. 2014;5(6).

42. Sakanaka A, Kuboniwa M, Takeuchi H, Hashino E, Amano A. Arginine-ornithine antiporter ArcD controls arginine metabolism and interspecies biofilm development of Streptococcus gordonii. J Biol Chem. 2015;290(35):21185-21198

43. Cohen TS, Prince AS. Activation of inflammasome signaling mediates pathology of acute P. aeruginosa pneumonia. J Clin Invest. 2013;123(4):1630-1637.

44. Singer M, et al. The third international consensus definitions for sepsis and septic shock (Sepsis-3). JAMA. 2016;315(8):801-810

45. CLSI. Performance Standards for Antimicrobial Susceptibility Testing; Twenty-Fourth Informational Supplement. CLSI document M100-S24. Wayne, PA: Clinical and Laboratory Standards Institute; 2014.

46. Marigo I, et al. Tumor-induced tolerance and immune suppression depend on the C/EBPbeta transcription factor. Immunity 2010;32(6):790-802

47. Swamydas M, Lionakis MS. Isolation, purification and labeling of mouse bone marrow neutrophils for functional studies and adoptive transfer experiments. J Vis Exp. 2013;77(77):e50586.

48. Fischer S, et al. Using OrthoMCL to assign proteins to OrthoMCL-DB groups or to cluster proteomes into new ortholog groups. Curr Protoc Bioinformatics. 2011; Chapter 6:Unit 6.12.1-Unit 6.1219.

49. Oliveros JC. VENNY An interactive tool for comparing lists with Venn diagrams. http://bioinfogp.cnb.csic.es/tools/venny/ index.html. Accessed September 14, 2016.

50. Darling AE, Mau B, Perna NT. progressiveMauve: multiple genome alignment with gene gain, loss and rearrangement. PLoS One. 2010;5(6):e11147.

51. Alikhan NF, Petty NK, Ben Zakour NL, Beatson SA. BLAST Ring Image Generator (BRIG): simple prokaryote genome comparisons. BMC Genomics. 2011;12:402.

52. Stamatakis A, Hoover P, Rougemont J. A rapid bootstrap algorithm for the RAxML Web servers. Syst Biol. 2008;57(5):758-771.

53. Yates JR, Eng JK, McCormack AL, Schieltz D. Method to correlate tandem mass spectra of modified peptides to amino acid sequences in the protein database. Anal Chem. 1995;67(8):1426-1436.

54. Elias JE, Gygi SP. Target-decoy search strategy for increased confidence in large-scale protein identifications by mass spectrometry. Nat Methods. 2007;4(3):207-214.

55. Huang da W, Sherman BT, Lempicki RA. Systematic and integrative analysis of large gene lists using DAVID bioinformatics resources. Nat Protoc. 2009;4(1):44-57. 